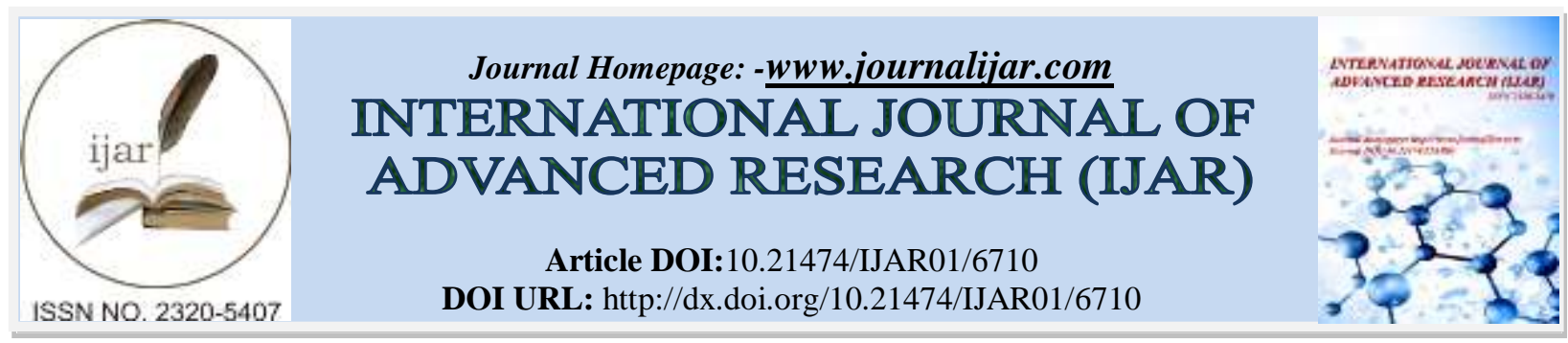

RESEARCH ARTICLE

\title{
MEDIAL PATELLOFEMORAL LIGAMENT RECONSTRUCTION: A COMPREHENSIVE REVIEW.
}

\section{Dr. Saravanan ${ }^{1}$ and Dr. Rajasekar ${ }^{2}$.}

1. MS ortho PG, Department of Orthopaedics, Sree Balaji Medical College and Hospital, BIHER, No.7, Works Road, New Colony, Chromepet, Chennai- 600044, Tamil Nadu, India.

2. Professor, Department of Orthopaedics, Sree Balaji Medical College and Hospital, BIHER, No.7, Works Road, New Colony, Chromepet, Chennai- 600044, Tamil Nadu, India.

\section{Manuscript Info}

Manuscript History

Received: 09 January 2018

Final Accepted: 11 February 2018

Published: March 2018

\begin{abstract}
In the majority of patellar dislocation cases, the medial patellofemoral ligament (MPFL) is disrupted with a high recurrence rate especially in female patients. Although MPFL tear is not the primary cause of instability, MPFL reconstruction is effective for stabilizing the knee and may alone prevent lateral patellar dislocation. There is limited but growing evidence that MPFL reconstruction for patellofemoral instability leads to excellent functional outcomes. Growing awareness of the biomechanical contribution of the MPFL has led to an upsurge in the publication of techniques and trials dealing with reconstructive techniques, warranting a review that includes the most recent evidence. The aim of this article was to review and summarize the recent literatures concerning MPFL reconstruction and provide a comprehensive review of previous studies ranging from basic science to current surgical techniques and results.
\end{abstract}

Copy Right, IJAR, 2018,. All rights reserved.

\section{Introduction:-}

Acute patellar dislocation is primarily an injury of active young patients of both sexes, with a higher recurrence rate in female patients1). The overall recurrence rate after primary patellar dislocation approaches 40\%2). Patients who have a primary patellar dislocation have a $17 \%$ recurrence rate, and patients who sustain repeated patellofemoral joint dislocation have a $49 \%$ recurrence rate.

There is growing interest in the soft-tissue structure that helps stabilize the patella. The medial patellofemoral ligament (MPFL) deficiency was reported to be $50 \%$ to $96 \%$ in those who had experienced traumatic patellar dislocation during open surgical exploration3). According to Amis et al.4), a rupture of this structure always occurs in lateral patellar dislocation because the MPFL can undergo a maximum elongation of 20\%-30\% (range, 18 to 20 $\mathrm{mm}$ ); this is far less than the patellar width, which often exceeds $40 \mathrm{~mm} 4$ ). The MPFL is often damaged during patellar subluxation or dislocation, and accordingly, over the last decade, several authors have recommended repair or reconstruction of the MPFL to reduce the high incidence of recurrent dislocation, and many different MPFL surgical reconstruction or repair techniques have been described in the literatures5). The aim of this article was to review recent literatures and provide a comprehensive review of anatomy, biomechanics, surgical techniques, radiologic anatomy, and clinical results. 


\begin{abstract}
Anatomy:-
In 1957, Kaplan6) first described the MPFL as the transverse reinforcement between the base of the patella and the tendon of the medial head of the gastrocnemius. Warren and Marshall's7) description of the three anatomic layers of the anteromedial region of the knee was the first to describe and name the MPFL and its location in the second layer between the medial epicondyle and the patella.The average length of the MPFL is $56.9 \pm 4.69 \mathrm{~mm}$ (range, 46.0 to $75.0 \mathrm{~mm}$ ), the mid-point width is $17.8 \pm 4.4 \mathrm{~mm}$ (range, 8.0 to $30.0 \mathrm{~mm}$ ), the width at the femoral insertion is $12.7 \pm 2.6 \mathrm{~mm}$ (range, 6.0 to $28.8 \mathrm{~mm}$ )8), and the thickness is $0.44 \pm 0.19 \mathrm{~mm} 9$ ). Lee et al.10) reported on MPFL anatomical measurements in Koreans: the width at the patellar attachment was $14.2 \mathrm{~mm}$ (range, 10 to $15 \mathrm{~mm}$ ), the width at the femoral insertion was $11.5 \mathrm{~mm}$ (range, 10 to $12.3 \mathrm{~mm}$ ), the average length of the upper border was 53.2 $\mathrm{mm}$ (range, 47.7 to $59.3 \mathrm{~mm}$ ) and that at the lower border was $55.4 \mathrm{~mm}$ (range, 51.0 to $59.7 \mathrm{~mm}$ ), and the thickness was $1.7 \mathrm{~mm}$ (range, 1.1 to $3.0 \mathrm{~mm}$ ) on the patellar side and $1.1 \mathrm{~mm}$ (range, 0.6 to $1.6 \mathrm{~mm}$ ) on the femoral side. The patellar attachment occupies $2 / 3$ of the proximal patella in $56.9 \%$ of cases, the proximal half in $41.2 \%$, the distal end in $1.3 \%$, and extends across the entire patella in $1.3 \% 8$ ).
\end{abstract}

The MPFL extends into the secondary layers below the deep fascia and superficial to the joint capsule along with the superficial band of the medial collateral ligament. Towards the femoral attachment, the MPFL passes beyond the limit of the joint capsule and then overlays the periosteum of the femoral condyle7). The patellar attachment is usually wider than the femoral attachment and is at the most prominent medial edge of the patella4).

The femoral attachment is located at $61 \% \pm 4 \%$ of the anteroposterior length from the anterior edge of the medial femoral condyle. The center of the anterior edge of the femoral attachment of the MPFL is, on average, $9.5 \mathrm{~mm}$ proximally and $5.0 \mathrm{~mm}$ posteriorly from the center of the medial femoral epicondyle9). The femoral attachment has been spread by decussating fibers that are attached to both the adductor tubercle and to the superficial fibers of the medial collateral ligament with more direct attachment to the epicondyle11).

The proximal insertion of the MPFL extends to the quadriceps tendon (Fig. 1). The patellar end of the MPFL passes deep to the distal vastus medialis obliquus (VMO), which overlays the MPFL at the patellar attachment and also attaches to the proximal part of the medial border of the patella4). The contact surface between VMO and MPFL has been reported as $20.3 \pm 6.0 \mathrm{~mm} 9$ ), $25.7 \pm 6.0 \mathrm{~mm}$, and $22 \mathrm{~mm}$ (range, 18.8 to $24 \mathrm{~mm}$ )10). There is a close relationship between the ligament and aponeurosis of the vastus intermedius and a loose connection with the VMO. The static structure of the MPFL contacts the tendon structures, creating an anatomical aponeurosis; during quadriceps contraction, this makes the entire system dynamic and is consequently crucial for medial patellofemoral joint stability. This anatomical aponeurosis guides and pushes the patella in the trochlea during active flexion, making it even more important to the integrity of the MPFL as a stabilizer8). This has a clear impact on clinical practice where proper muscular rehabilitation focuses on resolving maltracking or instability symptoms. When the vastus medialis contracts, the MPFL may be pulled proximally and become tighter. It is suggested that the VMO pulls the patella medially via the MPFL and acts not only as a direct dynamic stabilizer of the patella but also as an indirect dynamic stabilizer through the MPFL9).

Histology of the MPFL is not entirely clear, but macroscopically it resembles a tendon; however, from both anatomical and biomechanical viewpoints, it is undoubtedly a ligament8). 


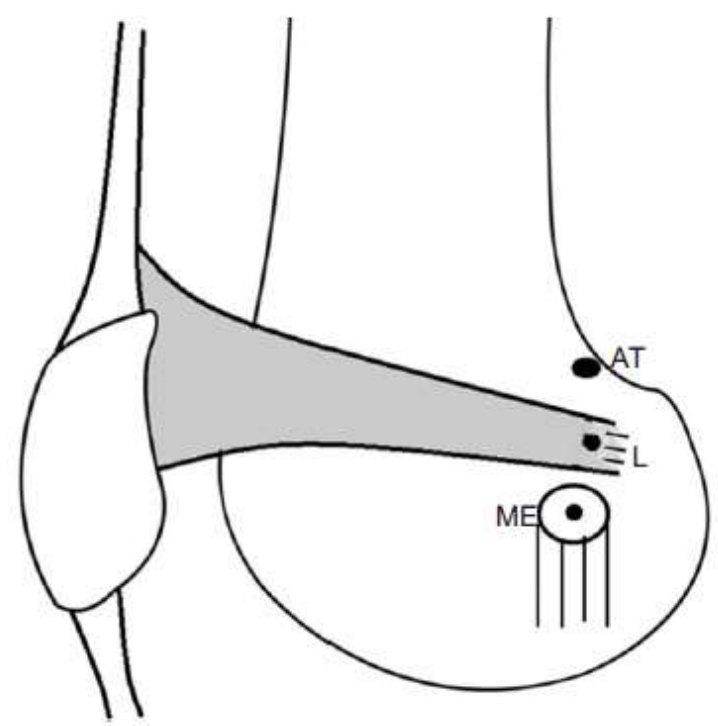

Fig. 1:-The relationship of the medial patellofemoral ligament (MPFL) to other structures. The patellar end of the MPFL passes deep to the distal vastus medialis obliquus (VMO), which overlays the MPFL at the patellar attachment. There is a close relationship between the ligament and aponeurosis of the vastus intermedius and a loose connection with the VMO. AT: center of the adductor tubercle,L: center of the anterior edge of the femoral attachment of the MPFL, ME: center of the medial femoral epicondyle.

\section{Biomechanics:-}

The patella is positioned within a soft-tissue sleeve extending from the anterior iliac spines of the pelvis and proximal femur to the tibial tubercle. Over the last $30 \mathrm{o}$ of knee extension, the patella lies outside the bony limits of the femoral trochlea, becoming more dependent on soft-tissue constraints12).

The MPFL provides approximately $60 \%$ of the total medial restraining force against lateral patellar displacement, whereas the medial patellomeniscal ligament, medial retinaculum, and medial patellotibial ligament contribute 13\%, $3 \%$, and 3\%, respectively, at 20o of knee flexion11). MPFL experiences maximal loads at full knee extension or during early flexion as quadriceps femoris neuromuscular activation pulls the patella toward the femoral trochATMELlea13,14). After 30o of knee flexion, the femoral trochlea contributes more to patellar stability. Nomura et al.9) reported that the MPFL was very taut at $0 \mathrm{o}$ of knee flexion, slightly relaxed at $15 \mathrm{o}-30 \mathrm{o}$ of knee flexion, and relatively taut at $45 \mathrm{o}-150 \mathrm{o}$ of knee flexion when a $1-\mathrm{kg}$ force was applied to the quadriceps tendon. Amis et al.4) reported that the patella could be subluxated laterally most easily at 20o flexion in the intact knee. The contribution of the MPFL to resisting patellar lateral subluxation was greatest in the extended knee.

Nomura et al.15) reported that the length change pattern was affected greatly if the femoral fixation point moved only $5 \mathrm{~mm}$ from the normal femoral attachment. Thus, the natural MPFL is clearly not isometric. Amis et al.4) reported that the length change pattern of a reconstructed MPFL depends critically on the site of femoral attachment, with a more proximal attachment increasing distance to the patellar attachment as the knee flexes; the converse is true for a distal attachment. This led to an experimental MPFL reconstruction that reproduced the two-bundled structure with a reciprocal tightening-slackening pattern as the knee flexes and extends.

The mean failure load was $208 \pm 90 \mathrm{~N}$ at $26 \pm 7 \mathrm{~mm}$ of displacement for specimens with a mean age of 72 years 16 ). Burks et al.17) noted a mean force of $209 \pm 55 \mathrm{~N}$ (range, 125 to $308 \mathrm{~N}$ ) at $25 \mathrm{~mm}$ of displacement for MPFL failure during lateral patellar dislocation.

\section{Surgical Technique:-}

Surgical Indication:-

Surgical treatment is generally recommended after a second dislocation. The MPFL reconstruction is most often used alone without a distal realignment or a trochleoplasty when the bony constructs are normal or near normal18), 
including trochlear dysplasia type A or normal trochlea, no trochlear spur, a tubero-sulcus angle of 0o to 10 o valgus, and no patella alta (Insall-Salvati ratio<1.4).

\section{Graft and Fixation:-}

The graft choice and fixation method is an important consideration. Considering the native MPFL resistance of around $200 \mathrm{~N}$, the graft choice should be a personal choice of the surgeon, since the commonly used grafts present satisfactory resistance. Case series with synthetic grafts, part of the adductor magnus tendon, semitendinosus, gracilis, part of the quadriceps tendon, or part of the patellar tendon and allograft all presented favorable results. Recently, many authors have reported using the gracilis tendon as a graft material19,20); this is because although the gracilis tendon is weaker than the semitendinosus tendon, it is still strong and has a stiffness closer to that of the native MPFL than that of the semitendinosus4).

The resistance to MPFL traction and similar methods of repair and reconstruction were studied by Mountney et al.16) who found a resistance of $208 \mathrm{~N}$ in the intact MPFL, $37 \mathrm{~N}$ with the isolated suture (Kessler method), $142 \mathrm{~N}$ with anchors associated with the sutures, $126 \mathrm{~N}$ with tendon grafts fixed in a blind tunnel, and $195 \mathrm{~N}$ with tendon grafts fixed in a bone tunnel through the femur from the medial femoral condyle to the lateral cortex.

There has been controversy regarding the proper knee flexion angle during graft fixation, with estimates ranging from 150 to 70o19-21). However, recent studies primarily recommend around 30o flexion20,22,23). Schottle et al.24) reported that graft fixation should be performed with the knee at $30 \mathrm{o}$ of flexion since biomechanical studies have shown that 30o flexion position is where the MPFL has its maximal restraint against patellar lateralization4). If the interference screw is used for the fixation in bone tunnel, the screw is turned into the tunnel until the lateral patellar edge is in line with the lateral femoral condyle edge, thus reconstructing the original anatomy of the MPFL. When the graft is fixed in the bone tunnel using an interference screw, the screwdriver is reversely rotated if the graft is overtightening during full range of motion of the knee joint, and patellar tracking is checked between 0 and 40 o of knee flexion, when the medial patellofemoral complex provides main restraint against patellar lateralization. If adequate medial restraint has been restored, lateral patellar dislocation should no longer be possible. It is important to avoid overcorrection, as it could lead to medial subluxation, graft failure, or medial patellofemoral pressure syndrome25). Feller et al.26) reported that graft tension should be adjusted to allow the patella to be displaced laterally within the one quadrant of the patella in 200 knee flexion.

Another important concern is the consequence of a positioning mistake when applying a graft during MPFL reconstruction. It is known that small mistakes of $5 \mathrm{~mm}$ from the ideal position or tensioning of the graft more than $2 \mathrm{~N}$ leads to an increase of the articular forces in the medial patellar facet, increasing the risk of pain and degeneration of the patellar cartilage15).

The authors' preferred graft choice is doubled gracilis tendon and preferred fixation method is suture anchor on the femoral attachment and multiple sutures on the periosteum of the patella. The authors' method is to ensure the lateral patellar edge to be in line with the lateral femoral condyle edge in $30 \mathrm{o}$ flexion position as described by Schottle et al.24) to avoid overtightening or loosening of the graft.

\section{Radiologic Landmarks for Femoral Insertion:-}

In 2007, Schottle et al.20), using plain radiographs, reported the radiologic landmark for the femoral origin of the MPFL is located between the medial epicondyle and the adductor tubercle. A reproducible anatomical and radiographic point, located $1 \mathrm{~mm}$ anterior to the posterior cortex extension line, $2.5 \mathrm{~mm}$ distal to the posterior origin of the medial femoral condyle, and proximal to the level of the posterior point of the Blumensaat line on a lateral radiograph with both posterior condyles projected in the same plane, shows the mean femoral MPFL center24) (Fig. 2). Stephen et al.27) reported that the MPFL attachment is defined in relation to the size of the medial femoral condyle: if the anterior-posterior size was $100 \%$, then the MPFL attachment was $40 \%$ from the posterior, $50 \%$ from the distal, and $60 \%$ from the anterior outline (Fig. 3). This point was most isometric, with a mean maximal length change to the central patellar attachment (center of proximal half of the patella) of $2.1 \mathrm{~mm}$ from $0 \mathrm{o}$ from $110 \mathrm{o}$ of knee flexion. The proximal femoral attachment resulted in mean lengthening of up to $6.4 \mathrm{~mm}$, and the distal attachment resulted in mean shortening of up to $9.1 \mathrm{~mm}$ through 0 to $110 \mathrm{o}$ of knee flexion, resulting in a significant non-isometry. Servien et al.28) reported that the points of femoral fixation had only $65 \%-69 \%$ of well-positioned 
tunnels, showing the difficulty of obtaining good positioning of the tunnels during surgery. They suggested a routine radiographic examination of the tunnel as often as possible.

Farrow et al.29) reported that the MPFL attachment was distal to the medial aspect of the femoral physis in all skeletally immature cadaveric specimens (Fig. 4). The MPFL attachment was an average of $8.5 \mathrm{~mm}$ distal to the medial aspect of the distal femoral physis. In the coronal plane, the undulating physis was $10.5 \mathrm{~mm}$ medial to the MPFL attachment. In all specimens, the undulations of the medial physis were concave directly posterior and lateral to the MPFL attachment and convex anterior to the MPFL attachment. Both the adductor tubercle and the medial epicondyle were also distal to the femoral physis in all specimens. These findings suggest that the femoral tunnel should not be drilled posteriorly or directly laterally because damage to the distal femoral physis may occur. Instead, angling the femoral tunnel distally and slightly anteriorly can help to avoid damage to the distal femoral physis during anatomic reconstruction of the MPFL in skeletally immature patients with recurrent patellofemoral instability.

Therefore, during MPFL reconstruction, the surgeon must avoid over-tension of the graft. It must be positioned without loosening and fixed in the anatomic position. The graft only becomes tense with the lateralization of the patella. Mistakes in positioning of the graft on the proximal-distal axis generate much more non-isometry than on the anterior-posterior axis. Changes of the patellar insertion exert a minor effect on MPFL isometry 15

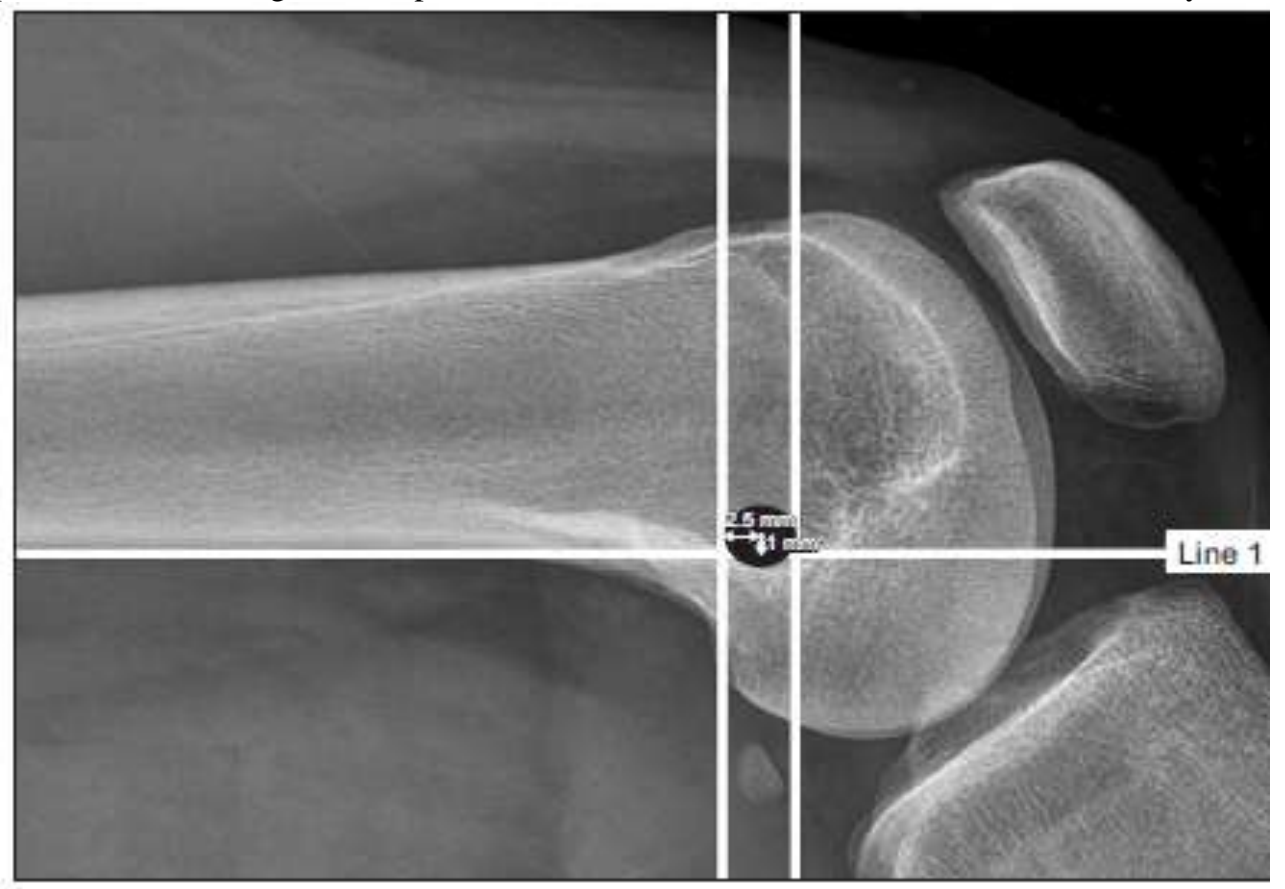

Fig. 2:- The Schottle's point at the center of the medial patellofemoral ligament (MPFL). Two lines perpendiculars to line 1 are drawn, intersecting the contact point of the medial condyle and the posterior cortex and intersecting the most posterior point of the Blumensaat line. This reproducible anatomical and radiographic point, located $1 \mathrm{~mm}$ anterior to the posterior cortex extension line, $2.5 \mathrm{~mm}$ distal to the posterior origin of the medial femoral condyle, and proximal to the level of the posterior point of the Blumensaat line on a lateral radiograph with both posterior condyles projected in the same plane, shows the mean femoral MPFL center. 


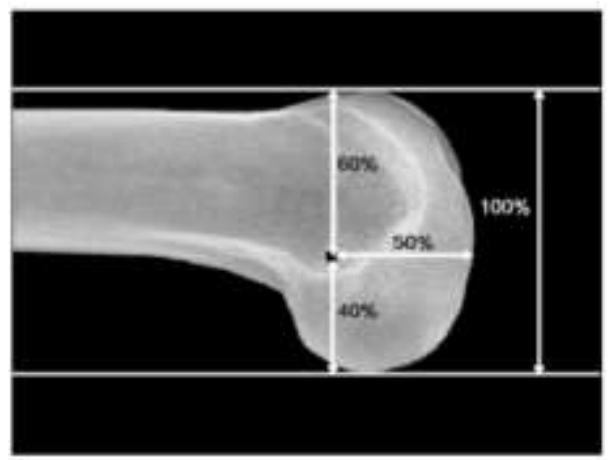

Fig. 3The medial patellofemoral ligament (MPFL) attachment is defined in relation to the size of the medial femoral condyle: if the anterior-posterior size is $100 \%$, then the MPFL attachment is $40 \%$ from the posterior, $50 \%$ from the distal, and $60 \%$ from the anterior outline.

\section{Clinical Results:-}

The first systematic review of MPFL reconstruction results was published in 2007 by Smith et al.30) and included eight papers with a total of 186 MPFL reconstructions. Despite favorable clinical and radiographic results, the critical analysis revealed methodological problems in the available published materials, including small samples, lack of control for confounding variables, absence of data concerning rehabilitation, works limited to case series, varied surgical techniques, and limited statistical analysis. Consequently, it was not possible to reach a consensus on the choice of graft, positioning, tensioning, or a preference between static and dynamic reconstruction. In 2010 , two more systematic reviews were published by Buckens and Saris5) and Fisher et al.31); their conclusions were similar to those of Smith et al.30).

Nomura et al.32) reviewed 22 former patients (24 knees) who had undergone MPFL reconstruction with a synthetic graft at a mean follow-up of 11.9 years. Lateral release was also performed in 14 knees. At final follow-up, CrosbyInsall scores included 11 excellent, 10 good, and 3 fair/poor. There were two cases of re-dislocation/subluxation. The mean Kujala score was 94.2 (from 63.2). The Crosby-Insall osteoarthritis classification was none-to-mild in 21 knees and moderate in 3 (from 23 and 1, respectively). This suggests the long-term superiority of MPFL reconstruction over older stabilizing procedures.

Watanabe et al.33) also compared MPFL reconstruction using a hamstring (semitendinosus or gracilis) tendon with tibial tubercle transfer (TTT) to reconstruction without TTT in 40 patients (42 knees) with recurrent patellar dislocation. This study also seems to demonstrate that MPFL reconstruction alone yields at least equally good results in patients with recurrent patellar dislocation at an average follow-up of 4.3 years.

Gomes34) compared two MPFL reconstruction techniques. He selected two matched-pair groups of 12 patients each for either adductor magnus rigid or semitendinosus dynamic femoral fixation. At a mean follow-up of 53 months, no statistical difference was detected between the groups despite one case of subluxation in the adductor magnus group. No major complication was observed in either group. Patients in the semitendinosus group felt subjectively better, and a larger number of patients in this group resumed the practice of sports. Even in the absence of significant differences, the present results suggest that a more dynamic femoral fixation is more advantageous than a rigid alternative.

Ronga et al.35) selected 28 patients with recurring instability without anatomic predisposition for treatment with MPFL reconstruction using a hamstring tendon (gracilis or semitendinosus tendon) fixed to the patella using transverse tunnels and to the medial epicondyle using a bone screw. MPFL reconstruction using hamstring tendon passed through a double patellar transverse bony tunnel is a safe, reliable management option for recurrent patellar dislocation in patients without any predisposing anatomic factors after an average of 3.1 years.

Enderlein et al.23) reported the largest MPFL reconstruction patient study to date. The study included 224 patients (240 knees) undergoing MPFL reconstruction. MPFL reconstruction with a gracilis tendon autograft consistently normalized patellar stability and improved knee function. Moderate medial pain was seen in $30 \%$ of the patients. Predictors of a poor subjective outcome included age over 30 years, obesity (body mass index $>30 \mathrm{~kg} / \mathrm{m} 2$ ), grade 3 or 4 cartilage injury, and female sex. 


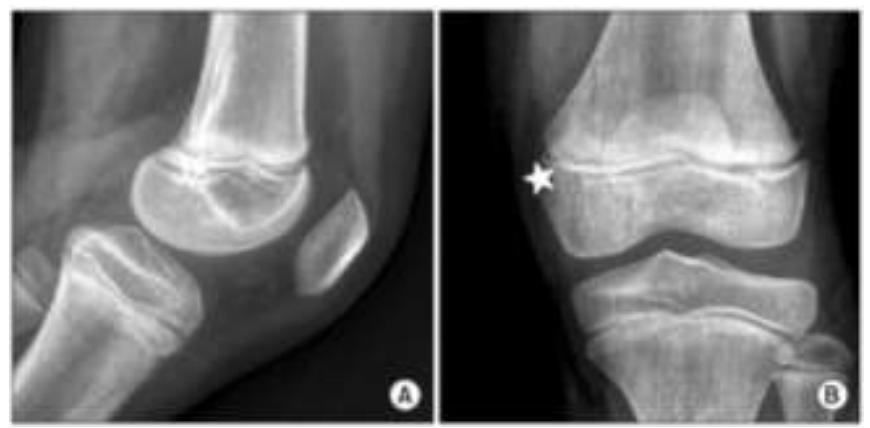

Fig. 4:-The medial patellofemoral ligament attachment is distal to the medial aspect of the femoral physis in skeletally immature patients even though it is observed on or proximal to the physis on the lateral radiograph. Crossreference of the physis on the lateral view (A) and the anteroposterior (AP) view (B) shows that the same point (star) that is projected on or proximal to the physis on the lateral view is distal to the physis on the AP vie

There are several clinical studies that report high success rates of MPFL repair performed for acute and recurrent patellar dislocation36,37). In these studies, a lateral release was routinely performed as part of the procedure. There are, however, studies by Dainer et al.37) and others that show increased rates of recurrent lateral instability when a lateral release is added to MPFL repair38). The lateral retinaculum provides a tether to the patella as it "ascends the slope" of the lateral trochlear as the patella moves in a lateral direction. Bedi and Marzo39) reported on the biomechanics of MPFL repair followed by lateral retinacular release. They concluded that after repair of the MPFL, adding a lateral retinacular release (LRR) lowered the ability of the patella to resist lateral displacement. LRR may not be routinely appropriate as a part of the stabilizing procedure to address acute patellar dislocation when the MPFL is avulsed from the patella. The MPFL is a stabilizing structure in resisting laterally directed forces on the patella. LRR should be carried out only after careful consideration.

Nelitz et al.40) reported clinical outcomes in skeletally immature adolescents with recurrent lateral patellar dislocation. Twenty-one consecutive patients with patellofemoral instability and open growth plates underwent anatomic reconstruction of the MPFL using gracilis tendon that maintained the distal femoral growth plate. The average age at the time of operation was 12.2 years (range, 10.3 to 13.9 years). The average follow-up after operation was 2.8 years (range, 2.0 to 3.6 years). No recurrent dislocation occurred, but two patients with high-grade trochlear dysplasia still had a positive apprehension sign. The Kujala score significantly improved from 72.9 (range, 37 to 87 ) preoperatively to 92.8 (range, 74 to 100 ) postoperatively ( $\mathrm{p}<0.01$ ). The Tegner activity score decreased, but not significantly, from 6.0 (range, 3 to 9) preoperatively to 5.8 (range, 3 to 9) postoperatively ( $\mathrm{p}=0.48$ ). Anatomic reconstruction of the MPFL that respects the distal femoral physis in skeletally immature patients is a safe and effective technique for the treatment of patellofemoral instability and allows patients to return to sports without re-dislocation of the patella.

\section{Summary:-}

Primary repair of MPFL does not provide complete recovery of adequate tensile strength. The femoral attachment has been spread by decussating fibers that are attached to both the adductor tubercle and the medial epicondyle. The patellar attachment covers over $2 / 3$ of the proximal patella. The proximal insertion of the MPFL extends to the quadriceps tendon. This anatomical aponeurosis guides and pushes the patella in the trochlea during active flexion, making it even more important to the integrity of the MPFL as a stabilizer. The MPFL provides approximately $60 \%$ of the total medial restraining force against lateral patellar displacement.

Surgical treatment is generally recommended after a second dislocation. The MPFL is most often used alone without a distal realignment or trochleoplasty when the bony constructs are normal or nearly normal. Recently, many authors have reported using the gracilis tendon as graft material. The reason for this choice was that the gracilis tendon, despite being weaker than the semitendinosus tendon, is strong and has stiffness closer to the native MPFL than does the semitendinosus tendon. There has been controversy regarding the proper knee flexion angle during graft fixation. Recent studies have primarily recommended about $30 \mathrm{o}$ of flexion. Moreover, the tension should be adequately adjusted during MPFL reconstruction so that the lateral patellar edge is in line with the lateral femoral condyle edge. An overcorrection should be avoided, which increases the risk of pain and degeneration of the patellar cartilage. The radiologic landmark for the femoral origin of the MPFL using plain radiographs is located between the medial 
epicondyle and the adductor tubercle. Mistakes in positioning of the graft on the proximal-distal axis generate much more non-isometry than on the anterior-posterior axis. Changes of the patellar insertion exert a minor effect on MPFL isometry.

\section{Conclusions:-}

There is limited but growing evidence that MPFL reconstruction for patellofemoral instability leads to excellent functional outcomes.

\section{References:-}

1. Sillanpaa P, Mattila VM, Iivonen T, Visuri T, Pihlajamaki H. Incidence and risk factors of acute traumatic primary patellar dislocation. Med Sci Sports Exerc. 2008;40:606-11.

2. Maenpaa H, Huhtala H, Lehto MU. Recurrence after patellar dislocation: redislocation in $37 / 75$ patients followed for 6-24 years. Acta Orthop Scand. 1997;68:424-6.

3. Nomura E, Horiuchi Y, Inoue M. Correlation of MR imaging findings and open exploration of medial patellofemoral ligament injuries in acute patellar dislocations. Knee. 2002; 9:139-43.

4. Amis AA, Firer P, Mountney J, Senavongse W, Thomas NP. Anatomy and biomechanics of the medial patellofemoral ligament. Knee. 2003;10:215-20.

5. Buckens CF, Saris DB. Reconstruction of the medial patellofemoral ligament for treatment of patellofemoral instability: a systematic review. Am J Sports Med. 2010;38:181-8.

6. Kaplan EB. Factors responsible for the stability of the knee joint. Bull Hosp Joint Dis. 1957;18:51-9.

7. Warren LF, Marshall JL. The supporting structures and layers on the medial side of the knee: an anatomical analysis. J Bone Joint Surg Am. 1979;61:56-62.

8. Placella G, Tei M, Sebastiani E, Speziali A, Antinolfi P, Delcogliano M, Georgoulis A, Cerulli G. Anatomy of the Medial Patello-Femoral Ligament: a systematic review of the last 20 years literature. Musculoskelet Surg. 2014 Jul 6 [Epub]. http://dx.doi.org/10.1007/s12306-014-0335-y.

9. Nomura E, Inoue M, Osada N. Anatomical analysis of the medial patellofemoral ligament of the knee, especially the femoral attachment. Knee Surg Sports Traumatol Arthrosc. 2005;13:510-5.

10. Lee HS, Choi JY, Ha JK, Lee YS, Yoo JH, Kim MK, Kim JG. Anatomical reconstruction of the medial patellofemoral ligament: development of a novel procedure based on anatomical dissection. J Korean Orthop Assoc. 2011;46:443-50.

11. Desio SM, Burks RT, Bachus KN. Soft tissue restraints to lateral patellar translation in the human knee. Am J Sports Med. 1998;26:59-65.

12. Heegaard J, Leyvraz PF, Van Kampen A, Rakotomanana L, Rubin PJ, Blankevoort L. Influence of soft structures on patellar three-dimensional tracking. Clin Orthop Relat Res. 1994;(299):235-43.

13. Bicos J, Fulkerson JP, Amis A. Current concepts review: the medial patellofemoral ligament. Am J Sports Med. 2007; 35:484-92.

14. Feller JA, Amis AA, Andrish JT, Arendt EA, Erasmus PJ, Powers CM. Surgical biomechanics of the patellofemoral joint. Arthroscopy. 2007;23:542-53.

15. Nomura E, Horiuchi Y, Kihara M. Medial patellofemoral ligament restraint in lateral patellar translation and reconstruction. Knee. 2000;7:121-7.

16. Mountney J, Senavongse W, Amis AA, Thomas NP. Tensile strength of the medial patellofemoral ligament before and after repair or reconstruction. J Bone Joint Surg Br. 2005; 87:36-40.

17. Burks RT, Desio SM, Bachus KN, Tyson L, Springer K. Biomechanical evaluation of lateral patellar dislocations. Am J Knee Surg. 1998;11:24-31.

18. Arendt EA. MPFL reconstruction for PF instability: the soft (tissue) approach. Orthop Traumatol Surg Res. 2009;95(8 Suppl 1):S97-100.

19. Christiansen SE, Jacobsen BW, Lund B, Lind M. Reconstruction of the medial patellofemoral ligament with gracilis tendon autograft in transverse patellar drill holes. Arthroscopy. 2008;24:82-7.

20. Schottle PB, Romero J, Schmeling A, Weiler A. Technical note: anatomical reconstruction of the medial patellofemoral ligament using a free gracilis autograft. Arch Orthop Trauma Surg. 2008;128:479-84.

21. Nomura E, Inoue M. Surgical technique and rationale for medial patellofemoral ligament reconstruction for recurrent patellar dislocation. Arthroscopy. 2003;19:E47.

22. Becher C, Kley K, Lobenhoffer P, Ezechieli M, Smith T, Ostermeier S. Dynamic versus static reconstruction of the medial patellofemoral ligament for recurrent lateral patellar dislocation. Knee Surg Sports Traumatol Arthrosc. 2014; 22:2452-7. 
23. Enderlein D, Nielsen T, Christiansen SE, Fauno P, Lind M. Clinical outcome after reconstruction of the medial patellofemoral ligament in patients with recurrent patella instability. Knee Surg Sports Traumatol Arthrosc. 2014;22:2458-64.

24. Schottle PB, Schmeling A, Rosenstiel N, Weiler A. Radiographic landmarks for femoral tunnel placement in medial patellofemoral ligament reconstruction. Am J Sports Med. 2007;35:801-4.

25. Elias JJ, Cosgarea AJ. Technical errors during medial patellofemoral ligament reconstruction could overload medial patellofemoral cartilage: a computational analysis. Am J Sports Med. 2006;34:1478-85.

26. Feller JA, Richmond AK, Wasiak J. Medial patellofemoral ligament reconstruction as an isolated or combined procedure for recurrent patellar instability. Knee Surg Sports Traumatol Arthrosc. 2014;22:2470-6.

27. Stephen JM, Lumpaopong P, Deehan DJ, Kader D, Amis AA. The medial patellofemoral ligament: location of femoral attachment and length change patterns resulting from anatomic and nonanatomic attachments. Am $\mathrm{J}$ Sports Med. 2012;40:1871-9.

28. Servien E, Fritsch B, Lustig S, Demey G, Debarge R, Lapra C, Neyret P. In vivo positioning analysis of medial patellofemoral ligament reconstruction. Am J Sports Med. 2011;39:134-9.

29. Farrow LD, Alentado VJ, Abdulnabi Z, Gilmore A, Liu RW. The relationship of the medial patellofemoral ligament attachment to the distal femoral physis. Am J Sports30. Smith TO, Walker J, Russell N. Outcomes of medial patellofemoral ligament reconstruction for patellar instability: a systematic review. Knee Surg Sports Traumatol Arthrosc. 2007;15:1301-14.

30. Fisher B, Nyland J, Brand E, Curtin B. Medial patellofemoral ligament reconstruction for recurrent patellar dislocation: a systematic review including rehabilitation and return-to-sports efficacy. Arthroscopy. 2010;26:1384-94.

31. Nomura E, Inoue M, Kobayashi S. Long-term follow-up and knee osteoarthritis change after medial patellofemoral ligament reconstruction for recurrent patellar dislocation. Am J Sports Med. 2007;35:1851-8.

32. Watanabe T, Muneta T, Ikeda H, Tateishi T, Sekiya I. Visual analog scale assessment after medial patellofemoral ligament reconstruction: with or without tibial tubercle transfer. J Orthop Sci. 2008;13:32-8.

33. Gomes JE. Comparison between a static and a dynamic technique for medial patellofemoral ligament reconstruction. Arthroscopy. 2008;24:430-5.

34. Ronga M, Oliva F, Longo UG, Testa V, Capasso G, Maffulli N. Isolated medial patellofemoral ligament reconstruction for recurrent patellar dislocation. Am J Sports Med. 2009; 37:1735-42.

35. Ahmad CS, Stein BE, Matuz D, Henry JH. Immediate surgical repair of the medial patellar stabilizers for acute patellar dislocation: a review of eight cases. Am J Sports Med. 2000; 28:804-10.

36. Dainer RD, Barrack RL, Buckley SL, Alexander AH. Arthroscopic treatment of acute patellar dislocations. Arthroscopy. 1988;4:267-71.

37. Vainionpaa S, Laasonen E, Silvennoinen T, Vasenius J, Rokkanen P. Acute dislocation of the patella. A prospective review of operative treatment. J Bone Joint Surg Br. 1990;72: 366-9.

38. Bedi H, Marzo J. The biomechanics of medial patellofemoral ligament repair followed by lateral retinacular release. Am J Sports Med. 2010;38:1462-7.

39. Nelitz M, Dreyhaupt J, Reichel H, Woelfle J, Lippacher S. Anatomic reconstruction of the medial patellofemoral ligament in children and adolescents with open growth plates: surgical technique and clinical outcome. Am J Sports Med. 2013;41:58-63 